\title{
Academic research at a South African higher education institution: Quality issues
}

\author{
S. Schulze \\ Department of Further Teacher Education \\ University of South Africa \\ Pretoria, South Africa \\ e-mail:schuls@unisa.ac.za
}

\begin{abstract}
The aim of the research was to critically analyse how a university context influences the quality of academics' research output. Wenger's social theory of learning was used as theoretical framework. The investigation involved an ethnographic case study of the research culture at one college at the institution. Data collection was mainly by means of participant observation, interviews and document analysis. In the light of Wenger's theory, the findings revealed that certain institutional practices facilitated high-quality research. These included financial incentives, some training programmes and travel opportunities to interact with other researchers. Practices that inhibited the delivery of quality research related to lack of job security, research support and uninterrupted time as well as excessive institutional control. Training that was disconnected from research practice did not stimulate quality research.
\end{abstract}

\section{INTRODUCTION}

'As education researchers we have a particular obligation and opportunity to take a leading role in seeing that the research that is done is truly good research' (Hostetler 1995, 16). These words came to mind when the South African Minister of Education questioned the quality of some South African journals and the fact that most of these are not recognised internationally (Brits 2006, 6). Of the 255 South African research journals, only 23 reached the three international index databases (ISI), and about a third have never been referenced in international publications.

South Africa's Department of Education expects every academic to publish at least 1.25 articles annually in journals the Department has accredited. Institutions receive financial rewards for meeting this target, and are penalised for failing to meet it. According to personal communication by a research management officer, the performance of academics at the South African higher education (HE) institution at which this study took place is lower than the expected 1.25 articles per academic per year. Moreover, during 2005, five of the 11 previously white establishments had better research output from their academics than this institution. 
In view of the above, the university in question compiled a research plan, which was circulated to members of the College Research Committee. The plan indicates that the institution aims to raise its research profile to the increment of researchers rated by the National Research Foundation (NRF), and to become one of the top five research institutions in South Africa.

It is clear from the above that the institution needs to improve the quality of its research output. This article therefore reports on issues of research quality. The literature review indicates the complexity of defining and assessing quality in educational research. The characteristics of successful researchers as well as institutional influences on research quality are interpreted in view of Wenger's social theory of learning in communities of practice which is used as theoretical framework for the study. The article goes on to report on an investigation of how the university context at the institution influences the quality of academics' research output. It concludes by identifying key obstacles to the delivery of quality research that warrant further investigation or discussion.

In the next section, the complexity of defining and assessing research quality is analysed. In particular, this section addresses assessment criteria; the role of peer and participant recognition as an indicator of quality; and the role of values to assess quality.

\section{RESEARCH QUALITY AND ITS ASSESSMENT}

\section{The issue of assessment criteria}

It is not easy to define 'good' research (Christiansen and Slammert 2005, 1057; Wolter, Keiner, Palomba and Lindblad 2004, 517). One or two writers reject the very possibility of criteria, at least as conventionally understood (Hammersley 2005b, 1). Others differ. For example, Hammersley $(2005 \mathrm{a}, 1)$ states that there are at least two reasons that evaluation criteria may be of value to educational research: criteria may enhance the possibility of producing reliable findings; and with there being so many different approaches in educational research, there is a need to make explicit the criteria by which work within the different genres will be judged.

Criteria may include the following parameters: (1) technical, to determine whether the issue has been investigated appropriately; (2) methodological, to see if the issue has been investigated systematically; (3) substantive, to evaluate whether it contributes to knowledge; and (4) impact, to see if the research aids understanding of current concerns. Indeed, impact was added as a criterion in the Research Quality Framework of Australia's Department of Education, Science and Training for the evaluation of research quality (Yates 2005, 391). Impact is often assessed by the number of citations from the research work that other authors publish.

In a slight variation on the above, Hammersley $(2005 \mathrm{a}, 6)$ states that different sets of criteria were needed depending on what was being assessed. For example: Is the research report adequate? Are the findings valid? Have the research aims been 
reached? Apart from practical aims, research may aim to contribute knowledge on an issue or theoretical problem, describe only, provide explanations (using relevant theories) or formulate new theories (Yates 2005, 397).

Quantitative, qualitative and practice-based research also require different criteria for evaluation. Quantitative approaches require rigorous measurement and control of variables. Criteria to evaluate quality would include generalisability and replicability. However, Yates $(2005,397)$ emphasises that too much thinking takes this as the only model of good work.

The issue of the criteria by which qualitative research should be judged has been one on which qualitative researchers have not reached agreement. According to Hammersley $(2005 \mathrm{~b}, 1)$, criteria of evaluation cannot be about validity 'since to assume this is to adopt a foundationalist or realist position' which some authors, for example Scheurich (1997, 80-93), reject. 'At most all there can be is a list of considerations that it is agreed in local circumstances should be taken into account in judging qualitative work, a list that can serve as no more than a reminder and that is always open to revision in the process of being used - indeed, which only gains any meaning in that context' (Hammersley 2005b, 1).

In phenomenological research, interviews are often the main method of data collection. However, informants may not tell the truth; interview data may be 'incomplete' as compared to participant observation; and interview data is often heavily shaped by context, especially by the interviewer (Hammersley 2005b, 4-5; Scheurich 1997, 61-79). Hence, some researchers insist that the use of interviews should be avoided altogether. They have a higher regard for ethnographies that restrict the analysis to naturally occurring data obtained through observation and discourse analysis. It is extremely difficult to evaluate the quality of such an analysis. Moreover, ethnographers often make inferences based on gut feelings that were formed by participation in the culture of those they study. This indicates that the variety of approaches in qualitative research may each require its own set of criteria for judging its worth.

In addition to the above, 'there is wide agreement that practice-based research should be appreciated as needing different kinds of criteria' (Jean McNiff 2007, personal communication via e-mail). In this regard, Furlong and Oancea (2005, 8-15) differentiate between four dimensions of research quality, each with its own set of criteria: (1) Epistemic dimensions of research quality refer to traditional dimensions of quality such as methodological and scientific robustness. Evaluation criteria include trustworthiness, contribution to knowledge, explicitness in designing and reporting, propriety (conformation to legal and ethical principles) and paradigm-dependent criteria. (2) The technological dimension of the research quality refers to its value for use. In other words, how does it provide practitioners with facts, evidence and new ideas? Evaluation criteria include timeliness, fitness for purpose, accessibility, concern for enabling impact and operationalisability. (3) The capacity building and value for people dimension relates to the contribution the research makes to the collective and personal growth of practitioners and policy makers. Criteria include 
partnership and collaboration, plausibility from a practitioner's perspective, reflection and criticism, receptiveness and stimulation of personal growth. (4) The economic dimension of research quality relates to whether the money invested in a research project was well spent. Evaluation criteria include cost-effectiveness, marketability, audit ability, feasibility, originality and value-efficiency. Steadman (2005, 23-25) recommended adding 'desirability' as an economic sub-dimension and questioned 'cost-effectiveness' and 'value-efficiency' because these were difficult to assess. He concluded that an overall judgement of quality could be based on the level of transparency in the project's reporting.

\section{Peer and participant recognition}

The traditional way of evaluating research quality is by means of peer review (Langfeldt 2004, 51; Yates 2005, 394). Since the application of evaluation criteria relies on the knowledge, experience and judgement of the assessor it has been labelled biased and unreliable (in Langfeldt 2004, 52). Moreover, if peer review takes place in groups the evaluation will be affected by the group pressure/motivation or collective shrinking - no one performs).

Public recognition by the very groups that are being investigated is also sometimes used to indicate research quality. For example, in addition to a research output that has successfully undergone a formal quality assurance process, the University of Auckland (New Zealand) recognises the following as indicators of research quality for Mãori research (The Faculty of Education Research Development 2005, 2).

- Testimony by a scholar of acknowledged repute, either in New Zealand or elsewhere (the scholar may be an eminent kaumãtua [male elder or old person] or an academically credentialed expert)

- Invitation to present at an event, such as a hui (meeting), which is acknowledged as being of wide significance to the Mãori

- Wide acclaim by the Mãori beyond the original presentation (e.g. as evidenced by media reports, including Mãori media)

- Conferment of tribal honours for the contribution

Similar indicators are used for research quality for Pacific research.

\section{The role of values to evaluate research quality}

Regarding the role of values in indicating research quality, Hostetler $(1995,16-21)$ emphasises that good education research is not only a matter of sound procedures, but should also improve people's lives. He states: 'If we can get past the question of what is good, we must ask whether the good thing is good for these people, at this time, in this situation (Hostetler 1995, 20). In the USA, 'addressing national priorities' is also seen as an important indicator of research quality (Yates 2005, 394). 
The abovementioned contrasts with the views of Weber (in Hammersley 2005b, 2) who does not believe that social science should necessarily be geared toward political goals in that it aims at eradicating inequality of various kinds, improving the lives of people or serving the goals of education or some political cause. In fact, he believes that social research should be value-neutral. Practical values (other than the value of truth) should not shape the goals of inquiry even though they are needed to provide the value-relevant framework in terms of identifying which phenomena to study.

The above mentioned clearly indicates the difficulty of identifying quality research. However, few would disagree on the central role of knowledge and insight into the practices of proven researchers within academic communities for producing quality research. For this reason, Wenger's social theory of learning in communities of practice (1998) is particularly useful.

\section{THEORETICAL FRAMEWORK: WENGER'S SOCIAL THEORY OF LEARNING}

According to this theory, learning takes place through participation in socially situated practices, called communities of practice. This means that researchers learn how to do research through their research-related interaction with one another and with different researchers' work. A research community could be a research team, a group of colleagues who work together within a research unit or a group of researchers from different institutions or countries.

A research community is held together by their particular practice, hence the term communities of practice. 'Practice' is a way of talking about the shared historical and social resources, frameworks and perspectives that sustain research and is continuously redefined. 'Community' is a way of talking about the social configurations in which our research enterprise is defined as worthy of pursuit.

Wenger $(1998,73)$ identifies three elements which characterise a community of practice: (1) There is mutual engagement of participants in actions whose meanings they negotiate with one another. (2) There is negotiation of a joint enterprise. This creates relations of mutual accountability among participants. (3) There is development of a shared repertoire. For example, a research community shares language, documents, symbols, tacit conventions, rules of thumb, recognisable intuitions, perceptions, understandings, underlying assumptions and world views.

Wenger's communities have established a practice that recognises certain types of participation as competence. New members, called 'legitimate peripheral participants', are allowed participation to learn. The practice of the community creates the 'curriculum'. Lave and Wenger $(1991,21)$ warn that training programmes should not separate instructional settings from actual performance since this could split learners' ability to manage the learning situation apart from their ability to perform the skill. With time, newcomers change their way of doing things as they learn the practice. Their identities as practitioners develop accordingly. 
When organising itself, a community should consider the following elements: events (e.g. conferences that bring the community together); leadership (such as project leaders and networkers); connectivity (e.g. to discuss research issues and offer support); membership; learning projects (that explore knowledge, find gaps and define projects that can close the gaps); and artefacts (e.g. documents, tools and websites) (Wenger 2000, 230-232).

The success of organisations depends on their ability to design themselves as social learning systems. The three constitutive elements of learning systems are: communities of practice, boundary processes among these communities, and identities as shaped by our participation in these systems. For example, a HE institution has a learning system for research. The building blocks of this system comprise different communities of practice that includes academics, librarians, statisticians and editorial staff. Inside communities learning takes place because competence and experience need to converge for a community to exist. Boundaries connect different communities. Boundary interactions can create bridges if they are well coordinated, transparent and negotiable (Wenger 2000, 234). An identity (e.g. as a researcher) is a lived experience of belonging or not belonging to a community. A healthy identity will cross boundaries between communities and be open to new experiences. It is a vehicle for participating in the social world (Wenger 2000, 239). The idea of identity is strongly tied to motivation - the desire to become a better practitioner/researcher.

According to Wenger $(2000,243)$, the abovementioned view on learning systems implies: (1) Individuals should identify the communities they wish to belong to. Learning means being motivated to engage and contribute to their practices. (2) For communities, learning means refining practices and ensuring new generations of members. Communities of practice should balance core and boundary processes to enable deep learning in certain areas and be linked to other parts of the system. (3) Organisations should foster social learning systems, but they cannot fully own or control them. Rather, organisations should support the interconnected communities of practice and provide conditions for optimal participation. Lave and Wenger $(1991,55)$ warn that organisations should carefully organise their socio-cultural spaces into accessible places of activity; make essential knowledge available; coordinate participation within a community; handle conflicts and the motivation of participants. Organisations also need to carefully consider the impact of their policies on performance (Foote, Matson, Weiss and Wenger 2002, 290).

Wenger's theory is used to interpret the literature review that follows and thereafter the investigation of how the university context of one higher education institution influences the quality of academics' research output.

\section{CHARACTERISTICS OF SUCCESSFUL RESEARCHERS}

In groundbreaking work on the characteristics of successful researchers, Bland and Schmitz (1986) and Creswell (1986) identified the importance of in-depth knowledge 
in a research area, the mastering of fundamental methodological skills and advanced skills relevant to the researcher's area of investigation.

Successful researchers are highly socialised to the values and attitudes of research in the academic profession. They value autonomy and their academic freedom because they are intrinsically motivated by the challenge, creativity and problemsolving of the research itself. Many successful researchers pursue more than one project simultaneously. If one project stalls or fails, another may prove successful.

In accordance with Wenger's theory, prolific researchers are both externally and internally oriented. They are closely involved with internal management and curriculum decisions, among others. They also engage in professional interaction with peers outside their institution by attending national meetings and collaborating with colleagues. Although daily research work is conducted independently, research networks with professional colleagues are vital to effective performance and offer researchers a few thinking partners. This is a particular affirmation of Wenger's theory that indicated the supportive role of networking in communities of practice.

\section{INSTITUTIONAL INFLUENCES ON THE QUALITY OF EDUCATIONAL RESEARCH}

In the 1980s at the University of Michigan, in a monograph of the Center for the Study of Higher Education, the following was stated: 'The place of employment is the single best predictor of faculty scholarly productivity' (in Bland and Schmitz 1986, 27). The influences on the quality of research that were identified are still relevant:

\section{Quality of graduate training at the institution}

If graduate training is seen as the initial training of researchers, the quality of this training would affect the research knowledge and skills of researchers.

\section{Reward system}

It is important to recognise accomplishments. A system of reward enhances research productivity and quality. This includes monetary reward, managerial praise and public recognition (Bland and Schmitz 1986, 30).

Developing quality researchers raises issues around workload arrangement and the distribution of faculty budgets (Yates 2005, 396). Although academics are generally expected to do both teaching and research, faculty reward and support for one activity is often at the expense of the other.

\section{Institutional support}

Institutional support that enhances quality research relates to the following:

Enough time for research: Sufficient time is needed to do quality research. In this regard, authors have estimated that research time somewhere in the 30 to 40 
percent range is probably ideal (in Bland and Schmitz 1986, 26) or at least 20 percent (Creswell 1986, 90). Apparently, this has not yet been refuted. Sabbatical leave also enables researchers to focus on research activities without interruption.

Academics and managers are acutely aware of the auditing culture at higher education institutions. Constant auditing brings increased workload to manage the compliance paperwork and means less time for research. It also brings about stress which may be motivational for some but counterproductive for others (Yates 2005, 399).

Research policies: Some policies facilitate quality research. This includes a stated policy of academic freedom in the workplace. A mixture of teaching, research and service contributes to the best performance in research.

Financial support: Financial resources for research projects and financial support for external communication (travel to professional meetings, conferences or for visiting scholars) enhance quality.

Differentiated support for differing needs of faculty members: This includes assistance by administrative staff such as full-time research assistants, research committees to coordinate research, secretaries and computer resources (e.g. support with statistical analysis of quantitative data). Staff development activities that encourage quality research include mentoring of novices, academic writing workshops, extensive programmes relating to research development for academics (e.g. training in research methods), support for external communication (e.g. via meetings and conferences) and support for internal communication (e.g. seminars, and sponsoring a research report series).

\section{Institutional culture and climate}

A strong research culture at an institution and in departments encourages quality research. A research culture is formed by practices that include recognition and reward for quality research, the identification of models of good research processes and administrative support. To formulate policies accordingly, those that serve on research committees should be high-research performers.

The work climate is critical. The ideal climate includes autonomy with affiliation, as research is a communal activity. In line with Wenger's theory, the academic research community involves close-knit work groups and frequent meaningful contact between colleagues so that research networks may be formed. Although mentoring of novices is important, researchers with low productivity often hinder their more skilled colleagues with excessive demands for support. This leads to decreased productivity of the more skilled researchers.

Using the aforementioned as background, this article critically analyses how the university context (policies, plans and practices) at the institution where this research took place influences the quality of research output. The article will argue that although some practices enhance the quality of research, others are detrimental to the value of research outputs. 


\section{RESEARCH DESIGN}

\section{Context}

The research was conducted at a South African higher education institution during a time of transformation. The institution had merged with two others and management struggled to align working conditions. This caused feelings of a lack of job security, which were aggravated by internal restructuring. For example, the institution was divided into five new colleges, and a number of research institutes which had conducted research or offered research support were closed down. These research institutes were closed down if they had focused on offering research support to postgraduate students and staff at the expense of their own research outputs. Accordingly, the relevant institute of the department under investigation for this article was dissolved.

The higher education institution has the following management structure for research. A Vice-Principal, Executive Deans of the five colleges and Directors of research institutes provide strategic leadership. (The college at which this research took place houses two institutes.) The Research Directorate administers institutional research processes and implements decisions of a Senate Research Committee. The Senate Research Committee regulates research matters.

Within the colleges, research is managed by college research committees (CRCs). The functions of the $\mathrm{CRC}$ are to monitor research; monitor the research budget; oversee applications for conferences, research, and research and development leave; encourage NRF applications; manage applications for subsidising accredited journals and books; monitor ethics and plagiarism; and align research functions in the college.

\section{Method}

The research approach was an ethnographic case study since this was the most appropriate method to determine the research culture of a college at the abovementioned institution. In line with an ethnographic approach, data collection was mainly by means of participant observation of naturally occurring data over a period of two years. This was complemented by numerous informal interviews in the relevant college and department of which field notes were made.

Near the beginning of the research project, college management requested that academics submit individual research plans for the following three years. They were expected to indicate their plans with regard to research projects, publication of books and articles, conference attendance and the delivery of postgraduate student research. The aim was to identify researchers who needed assistance and those who could apply for NRF rating. The individual research plans of all the members of the relevant department were analysed. After eight months an e-mail questionnaire was circulated in one department to investigate the usefulness of the plans. In the same 
department, research colloquia were presented every six weeks. These were attended and field notes were made.

In addition to the above, all documents relevant for research were analysed. These included policy documents (the institution's research policy, policy on research funding, policy on ethics and plagiarism, and policy on publishing of journals); the institutional research plan and research development plan; minutes of all the meetings of the CRC over a period of two years; and various research reports within the relevant college.

Ethical issues were considered (e.g. reporting is to be anonymous). Trustworthiness considerations included that the research was conducted in the natural setting of academics' everyday work in the relevant department, college and institution, over a long time period, and with participant feedback and member checking on insights gained. For example, the Research Director of the relevant college was asked to critically evaluate if the report presented a fair and accurate picture of the research culture at the college.

In particular, the aim of the research was to comment, in light of Wenger's theory, on the possible influences of the university context on research quality. To this end, the discussion of the findings focuses on (1) institutional vision and goals of research and (2) support structures and resources. Areas that warrant further investigation are also identified.

\section{FINDINGS}

\section{Institutional vision, goals and objectives of research}

Although the institutional research policy is based on academic freedom (Policy on Research Ethics 2006, 1), it is also based on a vision towards the African university in the service of humanity (Research Policy 2006, 3). Thus the Institutional Research Plan (2007) indicates that research should develop knowledge, skills and values necessary for developing the African continent. Research priority areas that are identified include a diminishing science and technology human resource base in South Africa; African Renaissance Studies; Poverty and Development Studies; HIV and AIDS Social Studies; and Crime Injury and Violence.

It is recognised that researchers work as part of a community of scholars who determine the paradigm within which research is undertaken in a discipline. Hence 'what constitutes acceptable research is discipline and subject-specific ... It should be recognised that one kind of research is not 'better' or more scientific than another' (Research Policy 2006, 5). Peer review is seen as the only realistic criterion to evaluate research (Research Policy 2006, 9).

Moving towards a system of greater control, the Research Policy $(2006,11)$ mandates a research audit in colleges every three years. This is influenced by expected research outputs over a five-year period by academics on the different post levels (Professors are required to have 7 outputs, Associate Professors 6, Senior lecturers 5, and so on). Outputs include articles published in accredited journals, 
papers in refereed conference proceedings, chapters in books and books written for experts and, more recently, postgraduate students who have completed their studies successfully. Generally, academics saw these requirements as reasonable although some had problems with the fact that many prestigious overseas journals were not recognised by the Department of Education. Other influences on academics' motivation to reach expected targets related to uncertain future developments at the university and feelings of an erosion of job security. Added to this was a lack of a work ethic based on self-worth and self-fulfilment (Report by School of Graduate Studies: Minutes of CRC meeting 2006, 2-5).

As explained, academics were requested to submit individual research plans for the following three years. Analysis of the plans of the relevant department indicated that novice researchers from previously disadvantaged backgrounds set much higher targets than the expected outputs for their post levels. They thus set themselves up for disappointment and failure. Eight months after submission of the individual research plans, an e-mail questionnaire was circulated to all members of the relevant department to enquire (1) if they could remember what they had promised; (2) if they consciously planned their research accordingly; and (3) if they still had the same plans in mind. Novice researchers did not respond. Of the remaining staff members, four indicated that they could remember what they had planned. Of these, only one proven researcher indicated that research went according to plan. Another could not remember that she had submitted such a plan! For the others, new responsibilities or opportunities that arose or a sheer lack of time prevented them from keeping to original research plans.

\section{Institutional resources and support}

The primary purpose of the institution's research policy is 'to encourage quality research by providing an enabling environment in which researchers can flourish' (Research Policy 2006, 6). However, staff reported a 'lack of a supportive environment' and a 'lack of sufficient incentives to publish' as militating factors against the delivery of quality research (Report by School of Graduate Studies: Minutes of CRC meeting 2006, 2-5). The resources and support that are provided may be divided into five broad categories, namely financial support, infrastructure, time, collaboration and training (Research Policy 2006; Institutional Research Plan 2007). Each of these will be considered briefly.

\section{Financial incentives and support}

The university has a plough-back policy. Thus the institution has resolved to use research earnings generated from subsidised publications, graduation of Masters and Doctoral students as well as research grants received from various funding bodies exclusively for research support as follows:

- There is special support for designated groups (women, black and young researchers), staff who are elected to international management committees, 
national workshops and conferences, research fellows and research assistantships. For each of these, there are various requirements and restrictions (Policy on Grants for the Research Fund 2006).

- Active researchers may be awarded grants for conference attendance (Policy on Grants from the Research Fund 2006). These grants may be obtained biannually on condition that there is a research output of at least three articles in accredited journals plus three conference papers during the previous five years. This rule is wavered for researchers with an NRF rating or a research output of at least five articles in accredited journals during the previous five years. However, a large amount of research funding is not allocated each year. This may be influenced by the requirement of producing an accredited article on the particular paper that was presented, after attending conferences and before other grants are offered. Interviews with proven researchers indicate that this requirement tends to lead to endless follow-up reports that staff detests and that make them feel supervised. Hence, they described the system as one of 'punishment, not reward'. In more than one set of minutes of CRC meetings during 2006, staff dissatisfaction regarding this issue is noted, in particular with regard to the fact that the requirements for local conferences are similar to those for overseas conferences. It seems that researchers are not aware of the fact that there is some flexibility around awarding grants for research and conferences (Report by School of Graduate Studies: Minutes of CRC meeting 2006, 2-5).

- Apart from awarding grants, the institution rewards meritorious research in several ways (Research Policy 2006). This includes the fact that promotion to senior positions depends, inter alia, on a research record (Research Policy 2006, 7). A Chancellor's Prize is awarded every two years to stars. According to the policy, there is also monetary reward for meritorious research. However, in CRC minutes during 2006, the issue of merit rewards that were paid previously and subsequently stopped was raised. This fuelled the feeling by some staff members that their contribution to the university was not appreciated (Report by School of Graduate Studies: Minutes of CRC meeting 2006, 2-5).

- At the institution, a portion of the money that is earned from the state for research output is channelled to academics' personal research funds. These may be used for attending conferences, paying the membership fees of academic associations or buying resources to support research. Staff may also request that the money be paid out in cash although tax deductions would significantly influence the amount. Many academics experience the above as huge incentives to deliver quality research that may be published. However, uncertainty over the position of research outputs accumulating to older members of staff approaching retirement, some of whom are among the college's most productive researchers, impeded negatively on the continuous delivery of quality research by this group. Low subsidies for books or chapters in books and no subsidy for university textbooks 
also inhibited the delivery of quality research in this form (Report by School of Graduate Studies: Minutes of CRC meeting 2006, 2-5).

- In the Policy on Grants from the Research Fund $(2006,8)$, departments and centres are encouraged to apply to the Research Committee to be recognised as Academic Centres of Excellence (ACE). Reasons for establishing ACEs are to encourage researchers to exchange ideas for enhancing the quality of research, to improve the quality of postgraduate training of students and to improve the effective use of research funding. To qualify, a department should be outstanding in teaching and research, undertake interdisciplinary research projects and have NRF-rated researchers, among others. However, at the time of writing there was no ACE in the relevant college and only one in the institution. The role of these in stimulating quality research therefore seemed minimal. An ACE seems to be the result of rather than the reason for quality research.

- One of the objectives of the institution's Policy on Research Ethics (2006) is to promote quality and legitimacy of research. The policy document acknowledges that a lack of funding may limit opportunities for research, force cost-saving procedures and thus compromise the quality of research. In an interview with two proven researchers they described their frustration at the bureaucracy of getting funding for a huge community service research project they had planned. Eventually, the expected output of four articles published in accredited journals led them to abandon the project commenting: 'Poor funding equals poor research'.

\section{Infrastructure to support research}

Institutional infrastructure to support quality research includes offices with modern computer facilities, a well-resourced library with efficient staff and a Department of Language Services. However, the issue of a lack of support with quantitative research, in particular with the statistical analysis and interpretation of data, was noted in several CRC minutes. Although this was followed up by the Senate Research Committee, the issue was not resolved as some managers did not view this as the responsibility of the institution. In addition, the closing down of research institutes left staff without the research support in their immediate environment that they had become accustomed to. For some academics, in particular novices, this added to the feeling of powerlessness.

The institutional infrastructure also supports a number of scientific journals. This support includes monetary as well as quality assurance functions (Policy: Publishing of journals 2006, 1). The editor is responsible for maintaining the journals' quality. References are made to an international editorial board, a 'rigorous peer review process', the provision of editing and proofreading services, software to identify plagiarism (Policy: Publishing of journals 2006, 2-3), and the delivery of journals of a high technical quality. However, in numerous minutes of CRC meetings, it was noted that the University Press experienced a serious problem with the timeous 
publication of journals (of up to six months). The main causes identified were a lack of funding and staff. Editors who were also staff members did not receive additional remuneration for their work; were not generally provided with publication schedules; and did not seem to have the time or infrastructure to do the necessary processing of articles. Thus, a lack of recognition from the institution for the work of editors was identified as a factor inhibiting the delivery of quality research reports (Report by School of Graduate Studies: Minutes of CRC meeting 2006, 2-5).

\section{Lack of time}

A lack of uninterrupted time was one of the main problems expressed by proven researchers and others. This was influenced by teaching commitments and administrative overload, in particular that relating to an auditing culture 'that required the completion of countless forms and attendance of endless meetings'. Many participants believed that they spent 'probably half of their time' in meetings and on administrative issues of various kinds.

The university normally offered two special leave arrangements for the purposes of research, namely recess leave and long leave (sabbatical) - the latter being available to researchers in each six-year cycle. However, new conditions of service make no provision for research leave although this matter was noted with concern at CRC meetings. Special academic leave for research purposes would, in future, be under close supervision of the different chairs of departments.

\section{Research collaboration}

Although the institution recognised research as a communal activity, a lack of emphasis on group research was reported as a militating factor against quality research (Report by School of Graduate Studies: Minutes of CRC meeting 2006, $2-5$ ). This related to the fact that group outputs were not rewarded as one output per author but were shared equally among all concerned.

There was also uncertainty on the issue of co-authorship with students (Report by School of Graduate Studies: Minutes of CRC meeting 2006, 2-5). Promoters and supervisors who co-authored articles with students received financial rewards for the outputs. This practice was frowned upon and considered unethical by some departments. The issue is still under debate but may inhibit quality outputs through this avenue.

\section{Research training}

The institution recognises the importance of graduate training for school academics in research knowledge and skills. However, some proven researchers also indicated that their graduate training was poor. Being intrinsically motivated they struggled to obtain the necessary research skills through their own efforts. 'It was purely on my own' one stated. For others, the high quality of the mentoring they experienced facilitated their research journeys. 
In light of the significant role of graduate research training in supporting quality research, the institution's decision to mandate permission from an ethical committee for all student assignments that include participants is noted with concern (Policy on Research Ethics 2006). As a mega institution the bureaucracy of this would prevent the inclusion of practical assignments in students' training and thus limit its quality.

Regarding the research training of academic staff, 20 workshops on the identified needs of academics were arranged and funded during 2006 and 2007. The workshops focused on writing for publication; postgraduate supervision; evaluating dissertations and theses; the effective use of the Internet for research; action research: understanding the theory of action; selecting and planning articles for a dissertation and thesis; time management; performance management; leadership skills; and team building and interpersonal skills. Participant feedback indicated that the workshops were useful (Graduate Studies: Annual report 2006). Both novice and proven researchers attended the sessions although novices were unable to reach the targets of some workshops (e.g. finalising draft articles for publication).

Within the relevant department under focus, six-weekly departmental colloquia with a research focus were arranged. Observation indicated that these were not well attended by novice researchers. Some of them seemed to prefer to approach proven researchers on an individual basis for support. The institution's Research Plan (2006) and Research Policy (2006) indicate that experienced researchers should see it as their duty to mentor novices. However, proven researchers were generally reluctant to do so for three reasons: (1) they were struggling to find time for their own research responsibilities; (2) they were not rewarded for mentoring in any way; and (3) they did not want to promote a culture of dependency. The lack of an efficient mentoring system and a nurturing environment for developing researchers were thus identified as factors militating against delivering quality research by this group (Report by School of Graduate Studies: Minutes of CRC meeting 2006, 2-5).

\section{DISCUSSION OF FINDINGS IN THE LIGHT OF WENGER'S THEORY}

Wenger's (2000) theory implies that the currency of social learning systems is collegiality, reciprocity, expertise, contributions to practice, and negotiating a learning agenda. It is not affiliation to an institution, assigned authority or commitment to a predefined deliverable. The primary source of value creation lies in informal processes, such as conversations and brainstorming. The value of organisational processes thus lies in serving informal processes. Organisations should place emphasis on the meaningfulness of participation in research communities and the possibility of building identities as competent researchers. Organisations should work towards linking different communities that constitute learning systems, offering channels, shared discourses and technology platforms and providing coordination among practices.

In consideration of Wenger's theory, some organisational practices at the institution in question impact positively on research quality. These include financial incentives 
that motivate researchers, and the presentation of numerous workshops for research training, on condition that these are not separated from actual performance (e.g. workshops that aim at the finalisation of researchers' draft articles for publication). Travel opportunities to meet with other researchers locally and abroad support interconnected communities of practice, enrich the 'curriculum' and build identity. An infrastructure that includes an excellent library and editorial support also falls into the category of fostering a social learning system for research.

Plans that fail to stimulate quality research include the forming of ACEs and the requirement that academics draw up individual research plans for a number of years. Failure to meet the unrealistic objectives that novices set for themselves could have a further negative impact on their self-worth, motivation and identities as competent researchers. Such a system of control does not foster learning systems nor enhance the quality of research outputs.

Other factors at the institution also undermine research quality because it does not stimulate collegiality or support informal learning processes. These include a rapidly changing environment which offers little job security; disagreement between management and staff regarding grant requirements; and an inefficient infrastructure for research support with regard to some issues (e.g. insufficient support for journal editors, for addressing research design issues, for statistical analysis and interpretation of data or for group research).

Research worth is undermined by poor quality graduate training, especially on the part of novices from disadvantaged backgrounds. Wenger's theory indicates that the ideal work climate for facilitating quality research requires close-knit work groups and frequent meaningful contact between colleagues to form research networks. Departmental colloquia and college workshops support such a climate. However, novices often do not attend colloquia or reach workshop targets. Reasons for their absence may include the fact that such training is often separate from actual performance and thus of little practical use. Novices need to be involved in actual research projects as learning practitioners and made responsible for smaller parts of the projects. In this way they learn through experience while their identities develop accordingly.

Continuous transformation and an auditing culture at the relevant institution leave academics with little time for quality research. Foote et al. (2002) warn that organisations should be sensitive to the impact of their decisions on available time for participation in informal processes (e.g. conversations and brainstorming) that support research.

\section{CONCLUSION}

The institution at which this research took place needs to improve the quality of its research output. The quality of educational research is influenced by the individual personality factors of researchers including a work ethic based on self-worth and selffulfilment. These interplay with environmental factors at the institution. I maintain 
that at the relevant institution some practices enhance research value. However, other management practices are counterproductive for the optimal functioning of social learning systems and therefore inhibit the quality of its research, despite aims to the contrary. The fact that some of these issues (e.g. those related to support with research design and data analysis) have not been resolved after a long period of debate may be due to a lack of research experience by some managers. Those that serve on research committees should be high-research performers themselves.

The research has also highlighted some issues that need further investigation. Two examples include: (1) the mentoring of novices by means of participation in actual research projects under the guidance of competent researchers; and (2) the impact of the discontinuation of recess leave, intended for research purposes. Why the university is moving towards a system of greater control remains unanswered. Reasons may include that fact that continuous transformation has impacted negatively on work ethic in many cases.

It is hoped that consideration of the issues raised by this research may increase the research output at the institution and enhance its quality. Thus the education researchers at the institution may take a leading role in ensuring that the research that is done is truly good research.

\section{REFERENCES}

Bland, C. J. and C. C. Schmitz. 1986. Characteristics of the successful researcher and implications for faculty development. Journal of Medical Education 61 (1): 22-31.

Brits. E. 2006. SA navorsingsjoernale se gehalte wek kommer - verslag. Beeld 15 Mei 2006:6.

Christiansen, I. M. and L. Slammert. 2005. A multi-faceted approach to research development (I): Addressing the myths. South African Journal of Higher Education 19 (6): 1047-1061.

College of Human Sciences: School for Graduate Studies: Annual report for 2006.

Creswell, J. W. 1986. Concluding thoughts: Observing, promoting, evaluating, and reviewing research performance. In Measuring faculty research performance. New directions for institutional research, no.50, ed. J. W. Creswell. San Francisco: Jossey-Bass.

Foote, N., E. Matson, L. Weiss and E. Wenger. 2002. Leveraging group knowledge for highperformance decision-making. Organizational Dynamics 31 (3): 280-295.

Furlong, J. and A. Oancea. 2005. Assessing quality in applied and practice-based educational research. A framework for discussion. Oxford: Oxford University Department of Educational Studies.

Graduate Studies: Annual report. 2006. Minutes of College Research Committee meeting.

Hammersley, M. 2005(a). Assessing quality in qualitative research. ESRC TLRP seminar series: Quality in educational research. Birmingham: University of Birmingham.

—. 2005(b). Methodological disagreement and the problem of quality. ESRC TLRP seminar series: Quality in Educational Research. Birmingham: University of Birmingham.

Hostetler, K. 1995. What is 'good' research? Educational Researcher August/September 2005:16-21. 
Langfeldt, L. 2004. Judging quality. Research Evaluation 13 (1): 51-62.

Lave, J. and E. Wenger. 1991. Situated learning: Legitimate peripheral participation. Cambridge: Cambridge University Press.

Policy on Grants from the Research Fund. 2006. Pretoria: University Publishers.

Policy on Research Ethics. 2006. Pretoria: University Publishers.

Policy: Publishing of journals. 2006. Pretoria: SENREC.

Report by School of Graduate Studies: Minutes of College Research Committee meeting. 2006.

Research Policy. 2006. Pretoria: University Publishers.

Scheurich, J. J. 1997. Research method in the postmodern. Qualitative studies series: 3. London: The Falmer Press.

Steadman, S. 2005. Practically perfect? Excellence in practice-based educational research. A discussion paper for seminar 3 in the ESRC TLRP seminar series: Quality in Educational Research. Sussex: University of Sussex.

The Faculty of Education Research Development. 2005. Research and scholarly activities at the Faculty of Education. Definitions and examples of research evidence for annual research reporting and compilation of evidence portfolios. Auckland: The University of Auckland.

Wenger, E. 1998. Communities of practice: Learning, meaning, and identity. Cambridge: Cambridge University Press.

- 2000. Communities of practice and social learning systems. Organization 7 (2): 225-246.

Wolter, S., E. Keiner, D. Palomba and S. Lindblad. 2004. OECD examiners' report on educational research and development in England. European Educational Research Journal 3 (2): 510-526.

Yates, L. 2005. Is impact a measure of quality? Some reflections on the research quality and impact assessment agendas. European Educational Research Journal (4):391-403. 